\title{
GROWTH ESTIMATES FOR MEROMORPHIC SOLUTIONS OF HIGHER ORDER ALGEBRAIC DIFFERENTIAL EQUATIONS
}

\author{
SHAMIL MAKHMUTOV, JOUNI RÄTTYÄ, AND TONI VESIKKO
}

\begin{abstract}
We establish pointwise growth estimates for the spherical derivative of solutions of the first order algebraic differential equations. A generalization of this result to higher order equations is also given. We discuss the related question of when for a given class $X$ of meromorphic functions in the unit disc, defined by means of the spherical derivative, and $m \in \mathbb{N} \backslash\{1\}, f^{m} \in X$ implies $f \in X$. An affirmative answer to this is given for example in the case of UBC, the $\alpha$-normal functions with $\alpha \geq 1$ and certain (sufficiently large) Dirichlet type classes.
\end{abstract}

\section{INTRODUCTION AND MAIN RESULTS}

Let $\mathcal{H}(\mathbb{D})$ and $\mathcal{M}(\mathbb{D})$ denote the sets of analytic and meromorphic functions in the unit disc $\mathbb{D}=\{z \in \mathbb{C}:|z|<1\}$, respectively. For $n, N \in \mathbb{N}$, consider the $N$-th order algebraic differential equation

$$
\left(f^{(N)}\right)^{n}+\sum_{k=1}^{n} P_{k, N}(f)\left(f^{(N)}\right)^{n-k}=0
$$

where

$$
P_{k, N}(f)=\sum_{j_{0}=0}^{m_{k, 0}} \sum_{j_{1}=0}^{m_{k, 1}} \ldots \sum_{j_{N-1}=0}^{m_{k, N-1}} a_{k, j_{0}, \ldots, j_{N-1}} \prod_{\ell=0}^{N-1}\left(f^{(\ell)}\right)^{j_{\ell}}, \quad k=1, \ldots, n,
$$

with $a_{k, j_{0}, \ldots, j_{N-1}} \in \mathcal{H}(\mathbb{D})$ and $m_{k, j} \in \mathbb{N} \cup\{0\}$ for all $j=0, \ldots, N-1$ and $k=1, \ldots, n$. The case $N=1$ reduces to the first order equation

$$
\left(f^{\prime}\right)^{n}+\sum_{k=1}^{n} P_{k}(f)\left(f^{\prime}\right)^{n-k}=0
$$

where

$$
P_{k}(f)=\sum_{j=0}^{m_{k}} a_{k, j} f^{j}, \quad 1 \leq k \leq n \in \mathbb{N}, \quad m_{k} \in \mathbb{N} \cup\{0\},
$$

and $a_{k, j} \in \mathcal{H}(\mathbb{D})$ for all $k=1, \ldots, n$ and $j=0, \ldots, m_{k}$.

The main result of this study is a pointwise growth estimate for the spherical derivative of meromorphic solutions of (1.1). The method of proof does not depend on the underlying domain and can be performed on any set. The normal and Yosida solutions of algebraic differential equations similar to (1.1) and (1.3) have been studied extensively via techniques like, for example, the Lohwater-Pommerenke method [1, 3]. There are multiple existing results concerning normality conditions and the behaviour of the spherical derivatives of the solutions [1, 9, 13]. The method we employ allows us to consider solutions in classes which are strictly smaller than the class of normal functions.

Before stating the results, a word about the notation used. The letter $C=C(\cdot)$ will denote an absolute constant whose value depends on the parameters indicated in the parenthesis, 
and may change from one occurrence to another. We will use the notation $a \lesssim b$ if there exists a constant $C=C(\cdot)>0$ such that $a \leq C b$, and $a \gtrsim b$ is understood in an analogous manner. In particular, if $a \lesssim b$ and $a \gtrsim b$, then we write $a \asymp b$ and say that $a$ and $b$ are comparable.

Theorem 1. Let $n, N \in \mathbb{N}$ and $M_{\ell} \in \mathbb{N} \cup\{0\}$ such that

$$
M_{0} \geq \max _{k=1, \ldots, n} \frac{m_{k, 0}}{k}-2 \quad \text { and } \quad M_{\ell} \geq \max _{k=1, \ldots, n} \frac{m_{k, \ell}}{k}-1, \quad \ell=1, \ldots, N-1 .
$$

Then each meromorphic solution $f$ of (1.1) satisfies

$$
\prod_{\ell=0}^{N-1}\left(\left(f^{(\ell)}\right)^{M_{\ell}+1}\right)^{\#} \lesssim \sum_{k=1}^{n}\left(\sum_{j_{0}=0}^{m_{k, 0}} \sum_{j_{1}=0}^{m_{k, 1}} \cdots \sum_{j_{N-1}=0}^{m_{k, N-1}}\left|a_{k, j_{0}, \cdots, j_{N-1}}\right|^{\frac{1}{k}}\right) .
$$

In particular, if $\max _{k=1, \ldots, n} \frac{m_{k}}{k} \leq M_{0}+2$, then each meromorphic solution $f$ of (1.3) satisfies

$$
\left(f^{M_{0}+1}\right)^{\#} \lesssim \sum_{k=1}^{n} \sum_{j=0}^{m_{k}}\left|a_{k, j}\right|^{\frac{1}{k}}
$$

Theorem 1 has the following immediate consequence which deserves to be stated separately.

Corollary 2. Let $n, N \in \mathbb{N}, \max _{k=1, \ldots, n} \frac{m_{k, 0}}{k} \leq 2$ and $\max _{k=1, \ldots, n} \frac{m_{k, \ell}}{k} \leq 1$ for all $\ell=$ $1, \ldots, N-1$. Then each meromorphic solution $f$ of (1.1) satisfies

$$
\prod_{\ell=0}^{N-1}\left(f^{(\ell)}\right)^{\#} \lesssim \sum_{k=1}^{n}\left(\sum_{j_{0}=0}^{m_{k, 0}} \sum_{j_{1}=0}^{m_{k, 1}} \cdots \sum_{j_{N-1}=0}^{m_{k, N-1}}\left|a_{k, j_{0}, \cdots, j_{N-1}}\right|^{\frac{1}{k}}\right) .
$$

The product of the spherical derivatives on the left hand side of (1.6) appears in a natural way in the study of (weighted) normal functions [4, 5, 6, 8, 11, 15.

The second part of Theorem 1 gives arise to the question of when for a given class $X \subset$ $\mathcal{M}(\mathbb{D})$ and $m \in \mathbb{N} \backslash\{1\}, f^{m} \in X$ implies $f \in X$. An immediate observation is that, roughly speaking, this implication cannot be true if $X$ is sufficiently small and defined in terms of the spherical derivative. More precisely, for $f_{p}(z)=(1-z)^{-p}$ with $0<p<1 / m<1$ we have $f_{p}^{\#}(z) \asymp|1-z|^{p-1}$ and $\left(f_{p}^{m}\right)^{\#} \asymp|1-z|^{m p-1}$ as $\mathbb{D} \ni z \rightarrow 1$. This shows that $\left(f_{p}^{m}\right)^{\#}$ is essentially smaller than $\left(f_{p}\right)^{\#}$ when $z \rightarrow 1$, yet of course $f_{p} \in \mathcal{N}^{1-p} \subset \mathcal{N}$ for all $0<p<1$. Recall that for $0<\alpha<\infty$, the class $\mathcal{N}^{\alpha}$ of $\alpha$-normal functions is the set of functions $f \in \mathcal{M}(\mathbb{D})$ such that

$$
\|f\|_{\mathcal{N}^{\alpha}}=\sup _{z \in \mathbb{D}} f^{\#}(z)\left(1-|z|^{2}\right)^{\alpha}<\infty,
$$

and its subset $\mathcal{N}_{0}^{\alpha}$ of strongly $\alpha$-normal functions consists of functions $f \in \mathcal{M}(\mathbb{D})$ such that $f^{\#}(z)\left(1-|z|^{2}\right)^{\alpha} \rightarrow 0$ as $|z| \rightarrow 1^{-}$.

We next offer an affirmative answer to the question of when $f^{m} \in X$ implies $f \in X$ in the case of certain function classes. To do this, definitions are needed. An increasing function $\varphi:[0,1) \rightarrow(0, \infty)$ is smoothly increasing if $\varphi(r)(1-r) \rightarrow \infty$, as $r \rightarrow 1^{-}$, and

$$
\frac{\varphi(|a+z / \varphi(|a|)|)}{\varphi(|a|)} \rightarrow 1, \quad|a| \rightarrow 1^{-}
$$

uniformly on compact subsets of $\mathbb{C}$. For such a $\varphi$, a function $f \in \mathcal{M}(\mathbb{D})$ is $\varphi$-normal if

$$
\|f\|_{\mathcal{N}^{\varphi}}=\sup _{z \in \mathbb{D}} \frac{f^{\#}(z)}{\varphi(|z|)}<\infty
$$


and strongly $\varphi$-normal if $\frac{f^{\#}(z)}{\varphi(|z|)} \rightarrow 0$, as $|z| \rightarrow 1^{-}$. The classes of $\varphi$-normal and strongly $\varphi$-normal functions are denoted by $\mathcal{N}^{\varphi}$ and $\mathcal{N}_{0}^{\varphi}$, respectively.

Let $\omega \in L^{1}(0,1)$. The extension defined by $\omega(z)=\omega(|z|)$ for all $z \in \mathbb{D}$ is called a radial weight on $\mathbb{D}$. For such an $\omega$, denote

$$
\omega^{\star}(z)=\int_{|z|}^{1} \omega(s) \log \frac{s}{|z|} s d s, \quad z \in \mathbb{D} \backslash\{0\} .
$$

The Dirichlet class $\mathcal{D}_{\omega^{\star}}^{\#}$ consists of $f \in \mathcal{M}(\mathbb{D})$ such that

$$
\int_{\mathbb{D}} f^{\#}(z)^{2} \omega^{\star}(z) d A(z)<\infty
$$

where $d A(z)=r d r d \theta / \pi$ for $z=r e^{i \theta}$. Moreover, the Dirichlet class $\mathcal{D}_{\alpha}^{\#}$ consists of $f \in \mathcal{M}(\mathbb{D})$ such that

$$
\int_{\mathbb{D}} f^{\#}(z)^{2}\left(1-|z|^{2}\right)^{\alpha} d A(z)<\infty
$$

A function $f \in \mathcal{M}(\mathbb{D})$ belongs to UBC if

$$
\sup _{a \in \mathbb{D}} \int_{\mathbb{D}} f^{\#}(z)^{2} \log \frac{1}{\left|\varphi_{a}(z)\right|} d A(z)<\infty,
$$

where $\varphi_{a}(z)=(a-z) /(1-\bar{a} z)$.

With these preparations we can state our next result.

Theorem 3. Let $m \in \mathbb{N} \backslash\{1\}, 1<p<\infty, \varphi$ a smoothly increasing and $\omega$ a radial weight. Then the following statements are valid:

(i) $f^{m} \in \mathcal{N} \Rightarrow f \in \mathcal{N}$;

(ii) $f^{m} \in \mathcal{N}_{0} \Rightarrow f \in \mathcal{N}_{0}$;

(iii) $f^{m} \in \mathcal{N}^{\varphi} \Rightarrow f \in \mathcal{N}^{\varphi}$;

(iv) $f^{m} \in \mathcal{N}_{0}^{\varphi} \Rightarrow f \in \mathcal{N}_{0}^{\varphi}$;

(v) $f^{m} \in \mathcal{D}_{1}^{\#} \Rightarrow f \in \mathcal{D}_{1}^{\#}$;

(vi) $f^{m} \in \mathcal{D}_{\omega^{\star}}^{\#} \Rightarrow f \in \mathcal{D}_{\omega^{\star}}^{\#}$;

(vii) $f^{m} \in \mathrm{UBC} \Rightarrow f \in \mathrm{UBC}$.

The proofs of the cases (i)-(iv) are based on the so-called five-point theorems, named by the celebrated result for normal functions due to Lappan [7]. Such results exist also in the setting of meromorphic functions on the whole plane and are usually given in terms of Yosida and $\varphi$-Yosida functions, see [1, 12]. Therefore we may obtain analogues of the statements (i)-(iv) for those classes; details are left for interested reader.

The argument of proof used in (v)-(vii) uses [16, Theorem 2] due to Yamashita on meromorphic Hardy classes. This is not exclusive for the disc either and can be performed also on the plane. The argument yields the inequality

$$
\int_{\mathbb{C}} f^{\#}(z)^{2}\left(\int_{|z|}^{\infty} \log \frac{r}{|z|} \omega(r) d r\right) d A(z) \lesssim \int_{\mathbb{C}}\left(f^{m}\right)^{\#}(z)^{2}\left(\int_{|z|}^{\infty} \log \frac{r}{|z|} \omega(r) d r\right) d A(z)+1,
$$

valid for all meromorphic functions $f$ and radial weights $\omega$ on $\mathbb{C}$. This is an analogue of (vi) for $\mathbb{C}$. One natural choice for $\omega$ in this case is $\omega(z)=(1+|z|)^{-\alpha}$ for $1<\alpha<\infty$.

Let $0<\alpha<1$ be fixed. By Theorem 3(i) we know that if $f^{m} \in \mathcal{N}^{\alpha} \subset \mathcal{N}$, then $f \in \mathcal{N}$. For $0<\alpha<1$ and $m \in \mathbb{N} \backslash\{1\}$, write

$$
\beta_{\alpha, m}=\inf _{0<\gamma \leq 1}\left\{f \in \mathcal{N}^{\gamma}: f^{m} \in \mathcal{N}^{\alpha}\right\} .
$$


The function $f_{p}$ with $p=\frac{1-\alpha}{m}$ considered after Corollary 2 and Theorem 3(i) show that $1-\frac{1-\alpha}{m} \leq \beta_{\alpha, m} \leq 1$. The exact value of $\beta_{\alpha, m}$ is unknown.

Theorem $\left[3\right.$ shows that $f^{m} \in \mathcal{D}_{\alpha}^{\#}$ with $\alpha \geq 1$ implies $f \in \mathcal{D}_{\alpha}^{\#}$. Further, the function $f_{p}$ with $-\frac{\alpha}{2 m}<p \leq-\frac{\alpha}{2}$ shows that $f^{m} \in \mathcal{D}_{\alpha}^{\#}$ with $\alpha<0$ does not imply $f \in \mathcal{D}_{\alpha}^{\#}$. It is natural to ask what happens with the range $0 \leq \alpha<1$ ? We do not know an answer to this question.

We next aim for combining Theorems 1 and 3 in order to find a set of sufficient conditions for the coefficients of (1.1) that force meromorphic solution $f$ to belong to certain function classes. To do this, some more notation is needed. For $0<p<\infty$, the weighted growth space $H_{p}^{\infty}$ consists of $f \in \mathcal{H}(\mathbb{D})$ such that

$$
\|f\|_{H_{p}^{\infty}}=\sup _{z \in \mathbb{D}}|f(z)|(1-|z|)^{p}<\infty .
$$

Similarly, $H_{\varphi}^{p}$ consists of $f \in \mathcal{H}(\mathbb{D})$ such that

$$
\|f\|_{H_{\varphi}^{p}}=\sup _{z \in \mathbb{D}} \frac{|f(z)|}{\varphi(|z|)}<\infty .
$$

For $0<p<\infty$ and a radial weight $\omega$, the Bergman space $A_{\omega}^{p}$ consists of $f \in \mathcal{H}(\mathbb{D})$ such that

$$
\|f\|_{A_{\omega}^{p}}^{p}=\int_{\mathbb{D}}|f(z)|^{p} \omega(z) d A(z)<\infty .
$$

Note that the Hardy-Spencer-Stein formula yields

$$
\|f\|_{A_{\omega}^{p}}^{p}=p^{2} \int_{\mathbb{D}}|f(z)|^{p-2}\left|f^{\prime}(z)\right|^{2} \omega^{\star}(z) d A(z)+\omega(\mathbb{D})|f(0)|^{p}, \quad f \in \mathcal{H}(\mathbb{D}),
$$

by [14, Theorem 4.2]. This explains how the associated weight $\omega^{\star}$ raises in a natural manner.

The following result is an immediate consequence of Theorems 1 and 3 ,

Corollary 4. Let $n \in \mathbb{N}$ and $M_{0} \in \mathbb{N} \cup\{0\}$ such that $\max _{k=1, \ldots, n} \frac{m_{k}}{k} \leq M_{0}+2$, and let $\varphi$ be smoothly increasing.

(i) If $a_{k, j} \in H_{k}^{\infty}$ (resp. $a_{k, j} \in H_{k, 0}^{\infty}$ ) for all $j=0, \ldots, m_{k}$ and $k=1, \ldots, n$, then each meromorphic solution $f$ of (1.3) belongs to $\mathcal{N}$ (resp. $\mathcal{N}_{0}$ ).

(ii) If $a_{k, j} \in H_{\varphi^{k}}^{\infty}$ (resp. $\left.a_{k, j} \in H_{\varphi^{k}, 0}^{\infty}\right)$ for all $j=0, \ldots, m_{k}$ and $k=1, \ldots, n$, then each meromorphic solution $f$ of (1.3) belongs to $\mathcal{N}^{\varphi}$ (resp. $\left.\mathcal{N}_{0}^{\varphi}\right)$.

(iii) If $a_{k, j} \in A_{\omega^{*}}^{\frac{2}{k}}$ for all $j=0, \ldots, m_{k}$ and $k=1, \ldots, n$, then each meromorphic solution $f$ of $(1.3)$ belongs to $\mathcal{D}_{\omega^{*}}^{\#}$.

(iv) If

$$
\sup _{a \in \mathbb{D}} \int_{\mathbb{D}}\left|a_{k, j}(z)\right|^{\frac{2}{k}} \log \frac{1}{\left|\varphi_{a}(z)\right|} d A(z)<\infty
$$

for all $j=0, \ldots, m_{k}$ and $k=1, \ldots, n$, then each meromorphic solution $f$ of (1.3) belongs to UBC.

It is well known that in (iv) one may replace the Green's function $\log \frac{1}{\left|\varphi_{a}(z)\right|}$ by the term $1-\left|\varphi_{a}(z)\right|^{2}$ in the statement. This is due to the analyticity of the coefficients. 


\section{Proof of Theorem 1}

We first multiply (1.1) by

$$
\left(\left(M_{N-1}+1\right)\left(f^{N-1}\right)^{M_{N-1}} \prod_{\ell=0}^{N-2}\left(\left(f^{(\ell)}\right)^{M_{\ell}+1}\right)^{\prime}\right)^{n}
$$

to obtain

$$
\begin{aligned}
\prod_{\ell=0}^{N-1}\left(\left(\left(f^{(\ell)}\right)^{M_{\ell}+1}\right)^{\prime}\right)^{n}+ & \sum_{k=1}^{n} P_{k, N}(f)\left(f^{(N)}\right)^{n-k}\left(M_{N-1}+1\right)^{n}\left(f^{(N-1)}\right)^{M_{N-1} n} \\
& \cdot\left(\prod_{\ell=0}^{N-2}\left(\left(f^{(\ell)}\right)^{M_{\ell}+1}\right)^{\prime}\right)^{n}=0
\end{aligned}
$$

and then divide it by $\prod_{\ell=0}^{N-1}\left(1+\left|f^{(\ell)}\right|^{2\left(M_{\ell}+1\right)}\right)^{n}$ to get

$$
\begin{aligned}
\prod_{\ell=0}^{N-1}\left(\frac{\left(\left(f^{(\ell)}\right)^{M_{\ell}+1}\right)^{\prime}}{1+\left|f^{(\ell)}\right|^{2\left(M_{\ell}+1\right)}}\right)^{n}+ & \sum_{k=1}^{n} P_{k, N}(f)\left(M_{N-1}+1\right)^{k}\left(f^{(N-1)}\right)^{M_{N-1} k} \\
& \frac{\left(\prod_{\ell=0}^{N-2}\left(\left(f^{(\ell)}\right)^{M_{\ell}+1}\right)^{\prime}\right)^{n}\left(\left(\left(f^{(N-1)}\right)^{M_{N-1}+1}\right)^{\prime}\right)^{n-k}}{\prod_{\ell=0}^{N-1}\left(1+\left|f^{(\ell)}\right|^{2\left(M_{\ell}+1\right)}\right)^{n}}=0 .
\end{aligned}
$$

By reorganizing terms and taking moduli, we deduce

$$
\begin{aligned}
& \prod_{\ell=0}^{N-1}\left(\left(f^{(\ell)}\right)^{M_{\ell}+1}\right)^{\#} \leq\left(\sum_{k=1}^{n}\left|P_{k, N}(f)\right|\left(M_{N-1}+1\right)^{k}\left|f^{(N-1)}\right|^{M_{N-1} k}\right. \\
& \left.\cdot \frac{\prod_{\ell=0}^{N-2}\left(M_{\ell}+1\right)^{n}\left|f^{(\ell)}\right|^{M_{\ell} n}\left|f^{(\ell+1)}\right|^{n}}{\prod_{\ell=0}^{N-1}\left(1+\left|f^{(\ell)}\right|^{2\left(M_{\ell}+1\right)}\right)^{n}}\left|\left(\left(f^{(N-1)}\right)^{M_{N-1}+1}\right)^{\prime}\right|^{n-k}\right)^{\frac{1}{n}} \\
& \leq \sum_{k=1}^{n}\left|P_{k, N}(f)\right|^{\frac{1}{n}}\left(M_{N-1}+1\right)^{\frac{k}{n}}\left|f^{(N-1)}\right|^{\frac{M_{N-1} k}{n}} \\
& \cdot \frac{\prod_{\ell=0}^{N-2}\left(M_{\ell}+1\right)\left|f^{(\ell)}\right|^{M_{\ell}}\left|f^{(\ell+1)}\right|}{\prod_{\ell=0}^{N-1}\left(1+\left|f^{(\ell)}\right|^{2\left(M_{\ell}+1\right)}\right)}\left|\left(\left(f^{(N-1)}\right)^{M_{N-1}+1}\right)^{\prime}\right|^{\frac{n-k}{n}} \\
& =\sum_{k=1}^{n}\left|P_{k, N}(f)\right|^{\frac{1}{n}}\left(M_{N-1}+1\right)^{\frac{k}{n}} \prod_{\ell=0}^{N-2}\left(\left(\left(f^{(\ell)}\right)^{M_{\ell}+1}\right)^{\#}\right)^{\frac{k}{n}} \\
& \cdot\left(\frac{\left|f^{(N-1)}\right|^{M_{N-1}}}{1+\left|f^{(N-1)}\right|^{2\left(M_{N-1}+1\right)}}\right)^{\frac{k}{n}}\left(\prod_{\ell=0}^{N-1}\left(\left(f^{(\ell)}\right)^{M_{\ell}+1}\right)^{\#}\right)^{\frac{n-k}{n}} \text {. }
\end{aligned}
$$


It follows that

$$
\begin{aligned}
\prod_{\ell=0}^{N-1}\left(\left(f^{(\ell)}\right)^{M_{\ell}+1}\right)^{\#} \leq & \left(\sum_{k=1}^{n}\left|P_{k, N}(f)\right|^{\frac{1}{n}}\left(M_{N-1}+1\right)^{\frac{k}{n}} \prod_{\ell=0}^{N-2}\left(\left(\left(f^{(\ell)}\right)^{M_{\ell}+1}\right)^{\#}\right)^{\frac{k}{n}}\right. \\
& \left.\cdot\left(\frac{\left|f^{(N-1)}\right|^{M_{N-1}}}{1+\left|f^{(N-1)}\right|^{2\left(M_{N-1}+1\right)}}\right)^{\frac{k}{n}}\right)^{\frac{n}{k}} \\
\lesssim & \sum_{k=1}^{n}\left|P_{k, N}(f)\right|^{\frac{1}{k}}\left(M_{N-1}+1\right) \prod_{\ell=0}^{N-2}\left(\left(f^{(\ell)}\right)^{M_{\ell}+1}\right)^{\#} \\
& \cdot \frac{\left|f^{(N-1)}\right|^{M_{N-1}}}{1+\left|f^{(N-1)}\right|^{2\left(M_{N-1}+1\right)}}
\end{aligned}
$$

where

$$
\begin{aligned}
\left|P_{k, N}(f)\right|^{\frac{1}{k}} & \leq\left(\sum_{j_{0}=0}^{m_{k, 0}} \sum_{j_{1}=0}^{m_{k, 1}} \cdots \sum_{j_{N-1}=0}^{m_{k, N-1}}\left|a_{k, j_{0}, \ldots, j_{N-1}}\right| \prod_{\ell=0}^{N-1}\left|f^{(\ell)}\right|^{j_{\ell}}\right)^{\frac{1}{k}} \\
& \leq \sum_{j_{0}=0}^{m_{k, 0}} \sum_{j_{1}=0}^{m_{k, 1}} \cdots \sum_{j_{N-1}=0}^{m_{k, N-1}}\left|a_{k, j_{0}, \ldots, j_{N-1}}\right|^{\frac{1}{k}} \prod_{\ell=0}^{N-1}\left|f^{(\ell)}\right|^{\frac{j_{\ell}}{k}}
\end{aligned}
$$

Hence

$$
\prod_{\ell=0}^{N-1}\left(\left(f^{(\ell)}\right)^{M_{\ell}+1}\right)^{\#} \lesssim\left(M_{N-1}+1\right) \sum_{k=1}^{n}\left(\sum_{j_{0}=0}^{m_{k, 0}} \sum_{j_{1}=0}^{m_{k, 1}} \ldots \sum_{j_{N-1}=0}^{m_{k, N-1}}\left|a_{k, j_{0}, \ldots, j_{N-1}}\right|^{\frac{1}{k}} I_{k, j_{0}, \ldots, j_{N-1}}(f)\right),
$$

where

$$
I_{k, j_{0}, \ldots, j_{N-1}}(f)=\prod_{\ell=0}^{N-1}\left|f^{(\ell)}\right|^{\frac{j_{\ell}}{k}} \prod_{\ell=0}^{N-2}\left(\left(f^{(\ell)}\right)^{M_{\ell}+1}\right)^{\#} \frac{\left|f^{(N-1)}\right|^{M_{N-1}}}{1+\left|f^{(N-1)}\right|^{2\left(M_{N-1}+1\right)}} .
$$

Therefore to deduce the assertion it suffices to show that $I_{k, j_{0}, \ldots, j_{N-1}}(f) \lesssim 1$. But a direct calculation shows that

$$
\begin{aligned}
& I_{k, j_{0}, \ldots, j_{N-1}}(f)=\prod_{\ell=1}^{N-1} \frac{\left(M_{\ell}+1\right)\left|f^{(\ell)}\right|^{\frac{j_{\ell}}{k}+M_{\ell}+1}}{1+\left|f^{(\ell)}\right|^{2\left(M_{\ell}+1\right)}} \frac{|f|^{\frac{j_{0}}{k}+M_{0}}}{1+|f|^{2\left(M_{0}+1\right)}} \\
& \leq \prod_{\ell=1}^{N-1}\left(M_{\ell}+1\right) \frac{\left(1+\left|f^{(\ell)}\right|^{2\left(M_{\ell}+1\right)}\right)^{\frac{\frac{m_{k, \ell}}{k}+M_{\ell}+1}{2\left(M_{\ell}+1\right)}}}{1+\left|f^{(\ell)}\right|^{2\left(M_{\ell}+1\right)}} \frac{\left(1+|f|^{2\left(M_{0}+1\right)}\right)^{\frac{\frac{m_{k, 0}}{k}+M_{0}}{2\left(M_{0}+1\right)}}}{1+|f|^{2\left(M_{0}+1\right)}} \\
& \leq \prod_{\ell=1}^{N-1}\left(M_{\ell}+1\right), \quad k=1, \ldots, n,
\end{aligned}
$$

by the hypotheses (1.5).

\section{Proof of Theorem 3}

We prove first (i). Assume on the contrary that $f \notin \mathcal{N}$. Then for each $Z \in f(\mathbb{D})$, with at most four possible exceptions, $\sup \left\{f^{\#}(z)\left(1-|z|^{2}\right): z \in \mathbb{D}, f(z)=Z\right\}=\infty$ by [7]. Let 
$Z \in f(\mathbb{D}) \backslash\{0, \infty\}$ be one of the points for which the supremum is infinity, and let $\left\{z_{n}\right\}_{n=1}^{\infty}$ denote a sequence of preimages of $Z$ such that $f^{\#}\left(z_{n}\right)\left(1-\left|z_{n}\right|^{2}\right) \rightarrow \infty$, as $n \rightarrow \infty$. Then

$$
\begin{aligned}
\left(f^{m}\right)^{\#}\left(z_{n}\right)\left(1-\left|z_{n}\right|^{2}\right) & =\frac{m\left|f\left(z_{n}\right)\right|^{m-1}\left(1+\left|f\left(z_{n}\right)\right|^{2}\right)}{\left(1+\left|f\left(z_{n}\right)\right|^{2 m}\right)} f^{\#}\left(z_{n}\right)\left(1-\left|z_{n}\right|^{2}\right) \\
& =\frac{m|Z|^{m-1}\left(1+|Z|^{2}\right)}{\left(1+|Z|^{2 m}\right)} f^{\#}\left(z_{n}\right)\left(1-\left|z_{n}\right|^{2}\right) \rightarrow \infty, \quad n \rightarrow \infty,
\end{aligned}
$$

and therefore $f^{m} \notin \mathcal{N}$. A reasoning similar to that in the case (i) with [2, Theorem 9] gives (iii) and (iv). Further, [3] together with Lappan's proof in [7] can be used to establish an analogue of the five-point theorem for strongly normal functions, which in turn gives (ii) as above.

To prove (v)-(vii), we use [16, Theorem 2]. It implies

$$
\int_{D(0, r)} f^{\#}(z)^{2} \log \frac{r}{|z|} d A(z) \lesssim \int_{D(0, r)}\left(f^{m}\right)^{\#}(z)^{2} \log \frac{r}{|z|} d A(z)+1, \quad 0<r<1 .
$$

By letting $r \rightarrow 1^{-}$we deduce

$$
\int_{\mathbb{D}} f^{\#}(z)^{2} \log \frac{1}{|z|} d A(z) \lesssim \int_{\mathbb{D}}\left(f^{m}\right)^{\#}(z)^{2} \log \frac{1}{|z|} d A(z)+1 .
$$

This together with the inequalities $1-t \leq-\log t \leq \frac{1}{t}(1-t)$, valid for all $0<t \leq 1$, yield $(\mathrm{v})$.

By integrating (3.2) over $(0,1)$ with respect to $\omega(r) r d r$ and applying Fubini's theorem we deduce

$$
\int_{\mathbb{D}} f^{\#}(z)^{2} \omega^{\star}(z) d A(z) \lesssim \int_{\mathbb{D}}\left(f^{m}\right)^{\#}(z)^{2} \omega^{\star}(z) d A(z)+\|\omega\|_{L^{1}(0,1)}
$$

from which the assertion (vi) follows.

By applying (3.3) to $f \circ \varphi_{a}$ we deduce (vii). This completes the proof of the theorem.

\section{REFERENCES}

[1] R. Aulaskari, S.A. Makhmutov and J. Rättyä, Weighted Yosida functions, Complex Variables and Elliptic Equations, Vol. 55 (2010), 167-172.

[2] R. Aulaskari and J. Rättyä, Properties of meromorphic $\varphi$-normal functions, Michigan Math. J. 60 (2011), 93-111.

[3] R. Aulaskari and H. Wulan, A Version of the Lohwater-Pommerenke Theorem for Strongly Normal Functions, Comput. Methods Funct. Theory 1 (2001), 99-105.

[4] H. Chen and P. Lappan, Products of spherical derivatives and normal functions, J. Austral. Math. Soc. Ser. A 64 (1998), no. 2, 231-246.

[5] H. Chen and P. Lappan, Normal families, orders of zeros, and omitted values, Ann. Acad. Sci. Fenn. Math. 21 (1996), no. 1, 89-100.

[6] J. Gröhn, On non-normal solutions of linear differential equations, Proc. Amer. Math. Soc. 145 (2017), no. 3, 1209-1220.

[7] P. Lappan, A criterion for a meromorphic function to be normal, Comment. Math. Helv. 49 (1974), 492495.

[8] P. Lappan, The spherical derivative and normal functions, Ann. Acad. Sci. Fenn. Ser. A I Math. 3 (1977), no. 2, 301-310.

[9] L. Liao, W. Su, C. C. Yang, A Malmquist-Yosida type of theorem for the second-order algebraic differential equations, J. Differential Equations, Vol. 187 (2003), 63-71.

[10] A. J. Lohwater, Ch. Pommerenke, On normal meromorphic functions, Ann. Acad. Sci. Fenn. Ser. A I No. 550 (1973), $12 \mathrm{pp}$.

[11] S.A. Makhmutov, $\alpha$-normal functions and Yosida functions, The Chuang special issue, Complex Variables Theory Appl. 43(3-4) (2001), 351-362.

[12] S.A. Makhmutov, Classes of meromorphic functions characterized by the spherical derivative, Soviet Math. Dokl. Vol. 33 (1986), No. 2, 450-455. 
[13] S.A. Makhmutov, Spherical Derivative of Meromorphic Solutions of Analytic Differential Equations, Differential Equations, Vol. 47 (2011), No. 2, 1-4

[14] J. A. Peláez and J. Rättyä, Weighted Bergman spaces induced by rapidly increasing weights, Mem. Amer. Math. Soc. 227 (2014), no. 1066.

[15] Y. Xu, Normal functions and $\alpha$-normal functions, Acta Math. Sin. (Engl. Ser.) 16 (2000), no. 3, 399-404.

[16] S. Yamashita, The meromorphic Hardy class is the Nevanlinna class, J. Math. Anal. Appl. 80 (1981), 298-304.

Department of Mathematics, College of Science, Sultan Qaboos University, P.O. Box 36, PC 123 Al Khodh, Muscat, Sultanate of Oman

E-mail address: makhm@squ.edu.om

University of EAstern Finland, P.O.Box 111, 80101 Joensuu, Finland

E-mail address: jouni.rattya@uef.fi

University of EAstern Finland, P.O.Box 111, 80101 Joensuu, Finland

E-mail address: tonive@uef.fi 